\title{
5 Steganography, the widely used name for data hiding
}

\begin{abstract}
Nowadays, global communication has no bounds. More information is being exchanged over some public channels that serve to be an important mode of communication. Without them, the field of technology seems to collapse. But awfully, these communications often turn out to be fatal in terms of preserving the sensitivity of vulnerable data. Unwanted sources hinder the privacy of the communication and may even temper with such data. The importance of security is thus gradually increasing in terms of all aspects of protecting the privacy of sensitive data. Various concepts of data hiding are hence into much progress. Cryptography is one such concept, and the others being watermarking and so on. But to protect the complete data content with some seamlessness, we incorporate concepts of steganography. It provides complete invisibility to any sensitive data that is being communicated. This prevents attracting unwanted attention from third-party sources, which helps to some extent with information safety. The field of big data is quite into fame these days as they deal with complex and large datasets. Steganographic methodologies may be used for the purpose of enhancing the security of big data since they also find ways of doing so.
\end{abstract}

Keywords: Steganography, Cryptography, Mean Squared Error, Peak Signal-to-Noise Ratio, Entropy

\subsection{Introduction}

The worldwide booming technological [1] trends bring along with it several disincentives, and overcoming that has become a challenging task. In these days modern research [2] has given a new dimension to almost all fields of technology. The new technologies have stressed on mediums being digital [3]. Communication [4] is the sole mediator between any sorts of technology that offers a service to mankind. Modern technologies are indeed a boon for the human race in many ways. Global network of computers serves as an error-free mediator in the source to destination delivery of any data/document [5]. Research is carried out at a rigorous level, and hundreds and thousands of newer techniques are coming up so as to sort complex day-to-day problems [6]. Technology has made life simpler and easier. But as it is said there is no rose without a thorn, so goes the way with these

Srilekha Mukherjee, Goutam Sanyal, National Institute of Technology, Durgapur, India. 
new technologies. It is often necessary to transmit essential and confidential information globally. They face many problems. Their original goal and progress is often hampered by few things, and security [7] is one such thing. Internet also acts as a major source or base to numerous fields that want to endow a critical grip while bracing communication. Information is being communicated or exchanged over the same.

We know that for technologies to reach and serve mankind, they are always braced with communication. Without communication no technology can reach any life. In these days, security is an extremely important issue [8] that has taken over the attention and concentration of many. Of course there are enough valid reasons of being so. The threat of data piracy has been a high risk factor for quite a long time. The mass reproduction of information is also a huge problem. This leads to security concerns [9], which became a major issue in communication. Modern communication requires certain measures to repulse the attention of any third party, which affirms immense secrecy along with full confidentiality of information. The field of data hiding [10] hence came to their rescue.

A communication is a two-way [11] process. The first way is where it is being packed and sends from the sender's side. The second is where the receiver receives and unpacks it. Now the receiver [12] is expected to receive the same exact package what the sender has actually packed. No problem arises if the aforesaid is the case and the receiver receives the same thing that was send by the sender. But the problem arises if the receiver does not receive the same packed thing that was send from the sender's side. Here lies the main disadvantage and flaw. Some third-party attacks [13] are being made in the way of these communications. These third parties tend to temper with the technological benefits from being achieved. The fullfledged benefits of technologies are made to be jammed. Hence, the purpose is not achieved or fulfilled. Therefore, it is extremely important to protect [14] the need of originality for the information being communicated.

\subsection{Security of information}

In today's highly dynamic and competitive world, information security serves to be the actual fuel for the engine of global communication. Information can be defined as processed data or facts. In order to safeguard its originality, several processing [15] has to be made before communication. The main motive in these cases is to communicate privately [16], although it is actually done via a public communicator medium.

These days, the concept of covert communication is increasing at an alarming rate. The safe end-to-end [17] delivery of data is the prime issue of concern in information security. Also, this has to be ensured without any interference. For the rest of the world, a seemingly unimportant and transparent communication takes place. 
Cryptography [18] is a well-known technique of data protection. In this technique, any data or information to be transmitted is first encoded, that is, it is converted to an encrypted [19] form. The main aim of cryptography lies in securing these contents in a possible way. This can be done with the help of some secret keys. Several policies of encryption, which completely facilitates data security, are actually effectuated. This objective is achieved by transforming the original data into an illegible form that cannot be understood by any eavesdropper [20] sitting in middle. By encrypted form, it is meant that it can be portrayed as any noisy form of the information. Any other form apart from the original one can be pointed as an encoded one. The output is actually an illegible one. It cannot be read or understood by any outsider. The original information is known as plain text [21] and the encoded information is known as cipher text [22]. Cryptography may be further classified as symmetric key cryptography and asymmetric key cryptography. The denoting difference is pointed out with the help of the keys used for encoding and decoding the information.

Shown next in Figure 5.1 are the types of cryptography that are used to encrypt information into some illegible form.

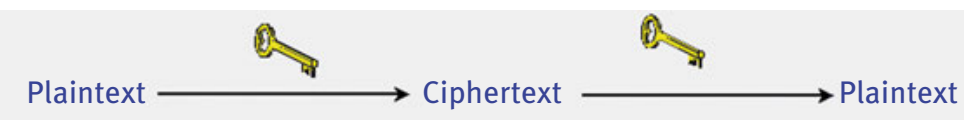

A) Secret key (symmetric) cryptography. SKC uses a single key for both encryption and decryption.

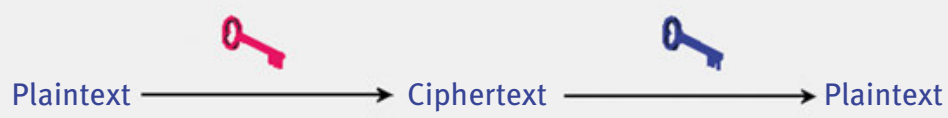

B) Public key (asymmetric) cryptography. PKC uses two keys, one for encryption and the other for decryption.

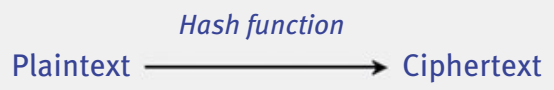

C) Hash function (one-way cryptography). Hash functions have no key since the plaintext is not recoverable from the ciphertext.

Figure 5.1: Types of cryptography.

\section{- Secret key cryptography}

These methods use a single key [23] for both encryption and decryption. The sender first uses this key for encrypting the necessary plaintext and generates the cipher text. The generated cipher text is then send to the concerned 
receiver. On receiving it, the receiver again uses the same key for decrypting the secret message. Thus, the receiver finally recovers the corresponding plaintext. A single key is applied for both encryption and decryption functions; therefore, secret key cryptography is again called as symmetric encryption. Basically, these methods may be applied and used for provision of confidentiality and privacy [24].

- Public key cryptography

These methods apply a single key for encryption purpose and one another for the purpose of decryption. Therefore, these methods are also known as asymmetric encryption/decryption. They can primarily be used for the purposes of authentication [25], key exchange, as well as nonrepudiation.

- Hash functions

Hash functions [26] are also known as message digests. They have one-way permitted encryption. Mainly these are algorithms, which generally use no key. Just a hash value of fixed length is estimated. This estimation is based on the plaintext. Also, this makes it nearly impossible for either the length of the plaintext or the contents to be recovered. The hash functions are most commonly used and applied by many operating systems for encrypting the entered passwords. Further, the hash functions provide a different mechanism to assure the integrity of a specific file.

Mainly, different methods or techniques of cryptography have been created for securing the secrecy of messages by encryption as well as decryption of data. The encrypted form of any data may often attract the attention of the third-party external sources. This makes it inquisitive for any unwanted external source and might intercept that this form may contain some hidden precious information from a source. This feature of cryptography may actually provoke any unintended third party about the covert communication that is being taking place. Hence, there arises an obvious requirement to hide any form of encryption made to protect and secure sensitive data.

Steganography [27] solves this issue by completely masking the information without making it visible to the outer sources. In order to avoid drawing any kind of suspicion, it has its methods to make some changes in the structures of the host so that it is not identifiable by any human eye. Therefore, the transmission of the hidden data is made in a completely undetectable manner. The communication in this case is completely kept hidden. It is a skill of hiding any confidential information within another media/entity such that nothing unusual appears in front of external sources. It hides the contents of data/any information within a carrier [28] medium, thus facilitating a seemingly invisible communication. Third parties are actually not able to see the information that is being communicated. Only the sender and receiver sides know and are aware about the secret communication being taking place. This particular advantage of steganography has increased its usage to a much higher level. It has given a new dimension to the concept of information 
security. The safety and integrity of sensitive data is guaranteed. All the fields and sectors have started using techniques that safeguards individual safety and security. Due to its immense potential of secured connectivity, it has become widespread. Therefore, the concepts of steganography are having huge demands in today's world. It facilitates privacy for several legitimate purposes during communication. Third parties are actually not able to see the information that is being communicated. Only the sender and receiver sides know and are aware about the secret communication being taking place. More communications takes place electronically in these days [29]. Likewise, for steganographic communications to take place, multimedia signals [30] are mostly chosen as renowned message carriers necessary for secured communication. There are many techniques that are figured out after highquality researches.

Another technique of watermarking [31] also has high usage in the field of information security. The main advantage in this case is to confirm the authenticity of any original data. Also, they may or may not be hidden in the host data. The watermark [32] is hidden within the host data in such a way that it possibly can never be removed. Even if its removal is made possible that can only be done at the cost of demeaning the concerned host data medium. Several watermarking applications, for example, copyright protection or source authentication may have an active adversary [33]. These stated groups may participate in making several attempts that removes, forges, or invalidates the embedded watermarks. Special inks have been used for hiding messages in the currencies as well. Steganography has its main goal of secure communication intact. The controlling factor is that the people are not by any chance aware of the presence of any hidden messages. This is what distinguishes steganography from any other forms of data hiding or information security.

\subsection{Steganography}

Steganography might be defined as the science and art of hiding data or information within another information, which appears to be harmless. The specific word "steganography" is a mere combination of two different Greek words, that is, "steganos" and "graphein," which means "covered" and "writing," respectively. The sensitive message can be hidden within a selected carrier known as the cover medium. This cover with the hidden data within is known as stego. The cover object serves to be any kind of medium within which any private message might be successfully embedded. This also aids to hide the presence of the very secret message, which is being sent. Referring to an image as a medium, we may say that the cover image is the seemingly unimportant image, within which the actual confidential image is to be embedded. On the other hand, the stego-image serves to be a carrier for communicating the private image across. 


\subsubsection{History of steganography}

Right from the ancient days [34], the concept of steganography had been used. The ancient kings and rulers used many techniques for data hiding. One was shaving the head of a trusted slave and then writing the message on his scalp. Once the hair grew back, he was sent to the corresponding recipient with that message. The recipient king shaved his head to read the message or information. A Greek historian Herodotus mentions a remarkable history related to this. Histiaeus, the chief of Miletus (an ancient Greek city), had sent a secret message to his concerning vassal, Aristagoras, the leader of Miletus, by shaving the head of one of his trusted servants. He then marked the secret message on the shaved scalp [35] and had sent him on his advised way when the hair on his scalp had regrown. This was one of the many techniques of how communication was made during those days.

Demaratus, the king of Sparta, from 510 until 491 BC had used this strategy to sent an anticipated warning for a forthcoming attack to Greece, by inscribing it directly on the underlying wooden support of some wax tablet. The final covering step was applying and smoothening its beeswax covered surface. Also, these waxcovered tablets were commonly used at that time as popular reusable writing surfaces. Even quite for some time, they were used for shorthand purposes.

Mary, Queen of Scotland, used to hide several letters with the combination of some techniques of cryptography and steganography. She had her secret letters hidden in a bunghole of some beer barrel that could freely pass in as well as out of her concerned prison cell. During World War II, another steganographic method that was practiced by the United States Marines was mainly the use of Navajo "code talkers.” They applied a kind of simple cryptographic technique and the messages used to be sent in all very clear text.

The vast uses of steganography were simply not limited to mere writing materials. The ancient use of large geoglyphs of the known Nazca lines in Peru can also be considered as a said form of the steganography. The figures vary in actual complexity. These geoglyphs are open to view, though most of them were not identified/detected until they were viewed directly from the above air. The designs are mainly shallow lines, which were made in the ground. It was done by removing the naturally existing reddish pebbles as well as uncovering the whitish or grayish ground underneath. Scholars have different opinion in interpreting their purposes. Moreover, in general, they accredit some sort of religious significance to those.

Another description of a human vector example does include writing secret messages on textures of silk. Later, this would be compressed and converted into one ball. A final covering with wax was the last step. The messenger then had to swallow this wax ball. In this case, the method for retrieving the secret message was not described in the sources.

Another example of steganography is the one that involves some specific use of the Cardano grille. Named after its very creator, Girolamo Cardano, this device 
can be considered to be as simple as a sample of paper with some holes made in it. The intended message can only be retrieved, when this grille is placed over some printed text. Such techniques might be related to the stated Cardano grille, which employs classical steganography techniques including methods of pin punctures in any text materials (e.g., newspapers) or overwriting some printed texts with any pencil.

Some evidences support that prior to the stated Civil War, there were certain methods of providing private messages to captured slaves that aided in their own escape. The quilts, which were mostly left to dry by hanging them from windowsills, were used as the target source. Secret messages were passed to the captivated slaves by some sort of patterns made in the quilts. This guided them in their venture for freedom. We may consider the Bear Paw symbol as an example of one such said quilt pattern. This represented an advice given to follow the found bear tracks over some of the region of mountains.

Some of the other uses of the stenographic techniques involve one photograph of the few captured crew members of the U.S.S. Pueblo. There all the crewmembers had spelled the same word "snowjob" and this was done using various hand positions. During the Vietnam era, some instances were found where during photo ops, the captured members in the U.S. Armed Forces would also use several hand gestures. This was often just to make these gestures aired by the media. The techniques mostly employed were by using their eyelids to blink some hints in Morse code, for example, torture. Also, the prisoners of the ill-famed Hanoi Hilton use to have a "tap code" for communicating among each other. This code was mainly based on a $5 \times 5$ matrix, where each of the residing letters is assigned a tap sequence. The sequence is purely based on the stated matrix. The spaces or pauses between those characters were twice as much long as the gaps or spaces in those particular letter codes.

There are many other examples from history, which relates to the same purpose as well. Use of invisible inks that glows when heated was one such example. Communication through microdots was one technique used during the World War days. There were certain other techniques as well.

\subsubsection{Modern steganography}

Digital communication is the boon of the trending technology. With the progression in this field of digital communication, the need of steganography serves to be a backbone of global communication. The need of security for the information traversed being the prime concern increases the demand of steganography. The sole reason was to secure and hide sensitive data from everyone except its intended recipient. This was the built-in feature of the domain steganography. It can actually guarantee the covert communication. This heightens the application range of steganography to an enormous extent. 
In the recent years, the global interest followed by research and development in this field has almost inflated to a high level. The presence of redundancy [36] in some of the representations of digital media (used as carrier or cover) is the targeted areas of data hiding in steganography. It attracted the attention of many researchers and developers, who decided to generate newer techniques of availing and sustaining covert communication [37]. During the communication stage, any unauthenticated people may only notice the transmission of a seemingly unimportant image.

A communication always takes place between two parties. One is the sender party and the other being the receiver party. Similarly in steganography, two processes take place: one in the sender side known as sender phase and the other at the receiver side, also known as receiver phase. Robustness as well as transmission security during communication are extremely essential for transmitting the vital message to its intended sources while declining access to unauthorized people. Hence, a secret point-to-point communication between the two trusted parties in the two sides should be ensured.

The communication channel is a public medium where any kind of untrusted source may be present. Their main aim might be mainly to uncover any secret data that passes by. Steganography evaluates and generates a number of ways until the attacker does not find some way to detect and trace the hidden information. With the communicating channel being selected, the communication proceeds with sending the stego. Many new techniques came up for the purpose of hiding information.

In these days communication has become digital. Therefore, the techniques used are digital steganographic techniques. A steganographic procedure has two phases: one taking place at the sender side and the other at receiver side. On the sender's side, the sender embeds the message within a chosen cover medium. On other hand, on the very receiver's side, the receiver extracts the hidden message from the received stego. The resemblance of the stego with its respective cover represents the efficiency of the procedure used. Also the efficiency of the algorithm lies in extracting the hidden information in a lossless manner. The lossy [38] extraction results in loss of data fields from the hidden information. This is definitely not what is expected out of a steganographic procedure, whose main aim is to communicate data secretly from sender to receiver. Therefore, if there is even a partial loss of hidden data, then the procedure is not fully efficient to what it promises.

\subsubsection{Benefits of steganography}

The primary demand of data integrity and authentication leads to absorption of certain effective measures in the respective systems. Government organizations have a wide range of use in this area. Various purposes of individual interests are some other important factors using the same. The self-conscience level of the modern crowd regarding the security attacks has increased a lot. People have become much more aware regarding protection of their personal and professional data. This self- 
awareness has led to an increase in the use of enhanced security in the communication systems. Even several trade and business purposes make use of the potential of steganography to communicate new product launching information or any other trade secrets.

\subsubsection{The major challenges of effective steganography}

The major challenges of effective steganography should be successfully met so as to achieve a potently secured communication. These requirements are for enabling of a secured communication. Therefore, for a good steganographic system, the following parameters should be significant.

- Robustness and security:

The term robustness [39] refers to resisting the attacks and securing the contents of the hidden data in a possible way. Thus, robustness is an important and challenging factor in any effective steganographic system. Also, for the facilitation of security, the data hidden must appear to be invisible to the external world. This concept of invisibility helps in achieving seamlessness in any carrier.

- Size of payload:

By the size of payload [40], we can say that this term is the total possible amount of secret data/message that can be hidden in any carrier or cover. The steganographic approach focuses in transmitting huge amounts of information maintaining the factor of imperceptibility. This amount of information hidden in any carrier should be maintained without breaking any of the other requirements (such as robustness and invisibility). Since steganography promotes hidden communication and therefore the requirements for higher payload along with secured communication are sometimes contradictory.

Note: It is always not possible to maximize capacity simultaneously with the trait of imperceptibility and improve robustness in any data hiding scheme. Henceforth, an acceptable balance of the above stated parameters must be sorted based on the necessary goal/application. For example, some steganographic schemes may forgo a bit of robustness in the favor of capacity with low perceptibility. On the other hand, a watermarking scheme, for which large capacity and low perceptibility is not a requisite, would definitely promote high robustness. Since the prime aim of steganography is hiding data, so the methods must promote sufficient capacity.

\subsubsection{Types of steganography}

Then, in Figure 5.2 the types [41] of steganography are based on the medium in which data is hidden. For example, if data is hidden in any text file, then it is text 
steganography [42]. If the cover medium is image, then it is image steganography and so on.

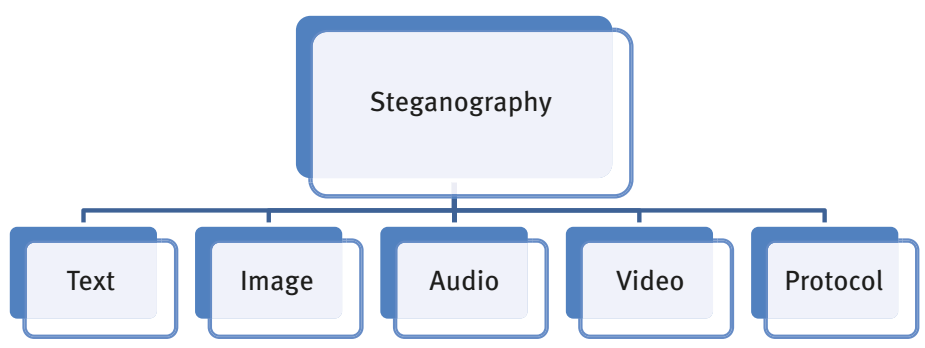

Figure 5.2: Types of steganography.

All these types are used in their relevant mediums, where hiding will be of utmost important in such medium. Various sectors of public importance emphasize in information hiding in the respective required medium, as per their necessity.

\subsubsection{Steganography using image as a medium}

Due to some subsistence of restricted potential of our human visual system [43], concealing information within digital images is asserted to be quite an efficient medium. Image steganography is also considered to be a potential for facilitating a secured communication globally.

\subsubsection{Types of image files}

There are two primary types of image files [44]: raster and vector (as specified in Figure 5.3). Any image may be catalogued in terms of either vector or any raster graphics. The image preserved in raster form is often said to be a bitmap. We may also define an image map as a file containing some information, which associates different locations on some given specified image with their hypertext links.

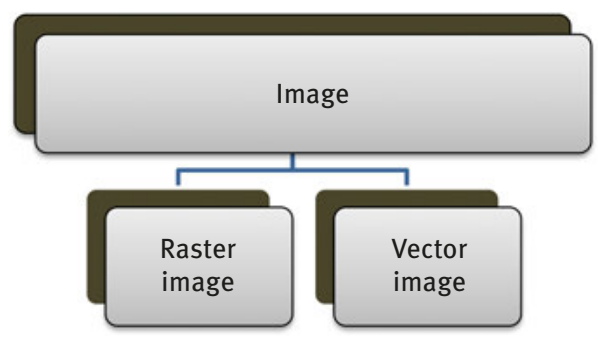

Figure 5.3: Two primary categories of image files. 


\section{- Raster image}

Raster images (Figure 5.4) are made up of collection of dots called pixels. These are generally more common (like PNG, JPG, GIF, etc.) and are widely used over web. Each pixel is specified as a tiny colored square. Suppose we zoom in to any raster image, we may see a lot of these little tiny squares. Raster images are the ones that are created with pixel-based programs. They may also be captured with a camera or scanner. When an image is scanned, it is converted to a collection of pixels, which we call a raster image.

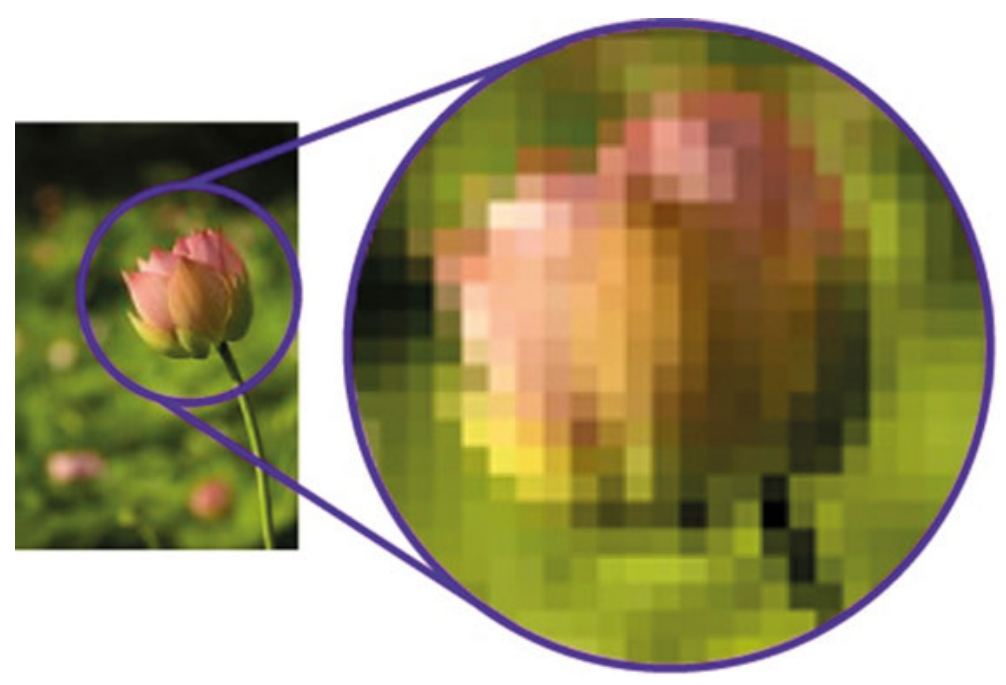

Figure 5.4: A raster image.

\section{- Vector image}

A vector image (Figure 5.5) is specified as one of the two major image file types. Vector graphics are those that are created with any vector software. These are more common for image files, which are applied onto any physical product. All vector images are object oriented while raster ones are pixel oriented. Since the vector graphics are not formed of pixels; therefore, they are resolution independent. Also, the vector shapes (called as objects) may be printed as large and at that highest resolution what the printer or output device allows. They always maintain all their details when zoomed in or out.

\subsubsection{Pixel}

A pixel [45] is denoted as a physical point present in a raster image. It is actually the smallest addressable element in any display device. 


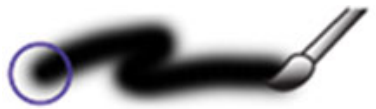

Painting with pixels
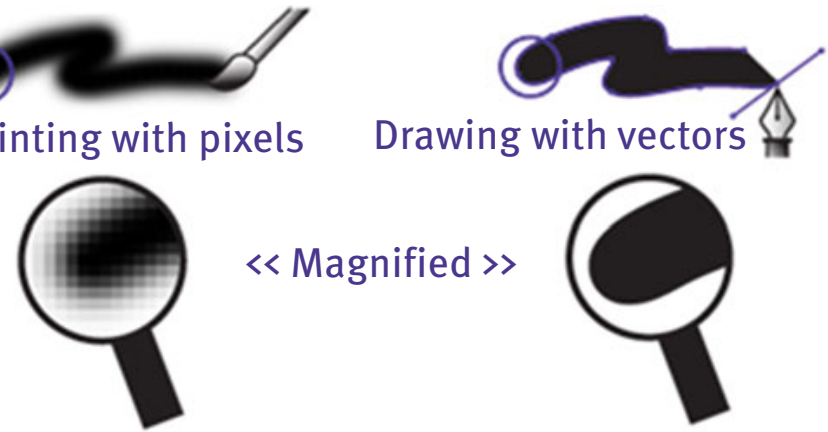

$<$ Magnified $\gg$

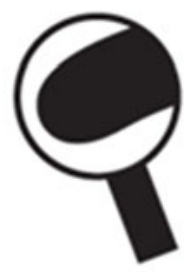

Figure 5.5: Rasters and vectors.

For example, in a $512 \times 512$ image that has 512 pixels considering side to side and 512 considering top to bottom has a total of $512 \times 512=262,144$ pixels.

\subsubsection{Types of image steganography}

There are several types of steganographic techniques that efficiently hide data. Broadly, it is categorized into two types of domain: spatial [46] and transform. Figure 5.6 shows few categories from both the spatial and transform [47] domain techniques.

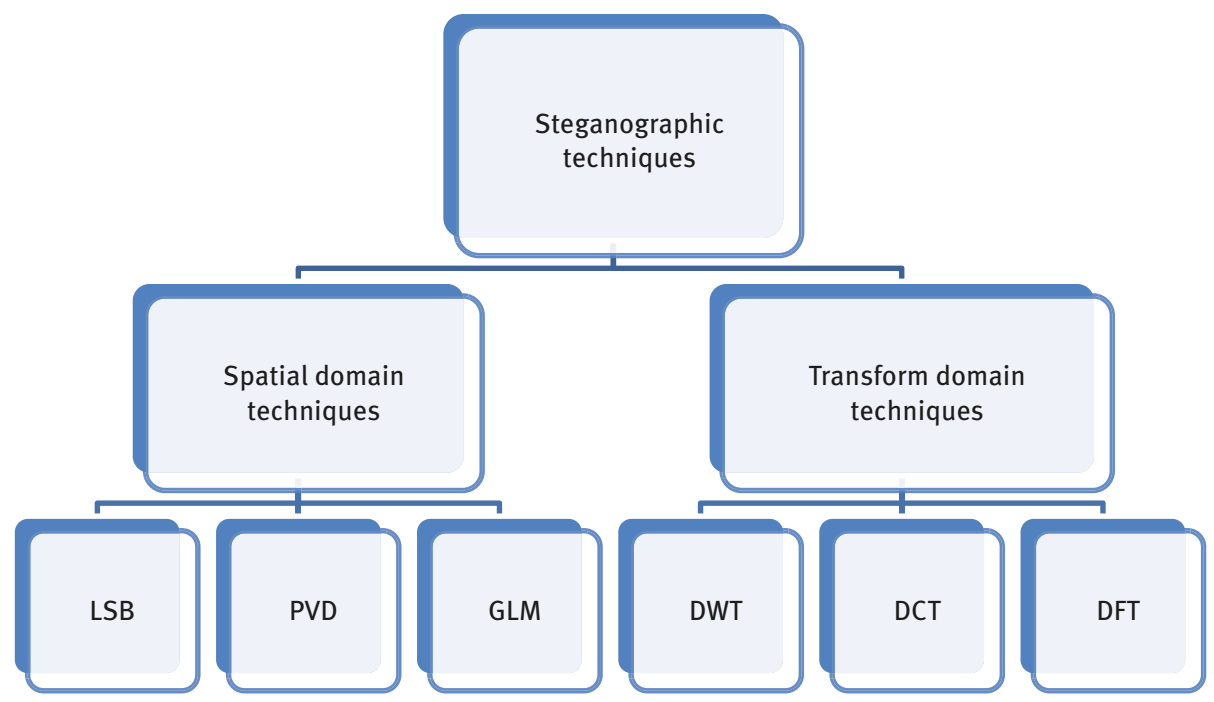

Figure 5.6: Domains in steganography. 
In the field of spatial domain, the secret data bits/messages are embedded directly into the cover bit planes. The least significant cover bits get directly replaced with the specific bits of secret message. There are a wide variety of procedures that use spatial domain techniques, like that of least significant bit [48] and pixel value differencing [49]. They are efficient in terms of several aspects like that of maximum data carrying capacity. In the transform domain, the secret message is embedded in their respectively transformed cover. There are a number of efficient transform domain techniques, like discrete wavelet transformation [50], discrete cosine transformation [51], and discrete Fourier transformation [52].

\subsubsection{Some analytic metrics}

There are several benchmark metrics based on which the efficiency of the constructed steganographic procedure may be found out. Accordingly, its strength can be determined. Given below are few such metrics, whose values may be computed and the efficiency of the steganographic output or rather stego may be found.

- Payload is stated as the total data-carrying capacity of that specific image, referred to as the carrier host or cover. The carried one is termed as the confidential or secret data. This carrier or confidential object might be any file, including some text, image, audio, and video. Henceforth, the embedding capacity of any cover or host image is the maximum capacity, which may denote that only on crossing this particular point distortion is recorded.

- Mean squared error or MSE: This is in correspondence to that expected value of the obtained squared error loss or the obtained quadratic loss. MSE [53] is actually a risk function. We may say that the difference occurs mainly due to the presence of randomness or may be the estimator do not account for any information that might generate a more accurate estimation. Thus, it supposedly incorporates both the variance of estimator along with its respective bias.

If we consider a cover image "CI" that has $M$ by $N$ pixels such that $M==N$ and a stego-image "SI $(i, j)$," which is obtained after hiding data within "CI," the MSE is found out as

$$
\mathrm{MSE}=\frac{1}{\left(M^{\star} N\right)} \sum_{i=1}^{M} \sum_{j=1}^{N}[\mathrm{CI}(i j)-\mathrm{SI}(i j)]^{2}
$$

- Peak signal-to-noise ratio (PSNR): While the MSE represents the obtained cumulative squared error measured between the images, the PSNR [54] represents a specific measure of the existing peak error between the stego and original. Now, for color images (i.e., having three RGB component values per pixel), the PSNR definition is same. It's just that the MSE is now calculated as the sum of 
all the squared value differences, which is divided by the respective image size and also by three. It is formulated as

$$
\operatorname{PSNR}=\frac{10 \log _{10}\left(255^{2}\right)}{\operatorname{MSE}} \mathrm{db}
$$

- Structural similarity index measure (SSIM): In general, SSIM [55] is considered to be a full reference metric. This signifies that the measure of any image quality depends on an uncompressed initial or rather distortion-free image, which is used as a reference. Now, we may say that this structural information is the primary idea that the resident pixels have very strong interdependencies. Also this is especially the case when they are close spatially. These kinds of dependencies always carry some relevant and important information related to the very structure of objects in their visual scene. It is calculated as

$$
\operatorname{SSIM}(c, s)=\frac{\left(2 \mu_{c} \mu_{s}+v 1\right)\left(2 \sigma_{c s}+v 2\right)}{\left(\mu_{c}^{2}+\mu_{s}^{2}+v 1\right)\left(\sigma_{c}^{2}+\sigma_{s}^{2}+v 2\right)}
$$

where $\mu_{c}$ is the mean of cover, $\mu_{s}$ is the mean of stego, $\sigma_{c}{ }^{2}$ is the variance of cover, $\sigma_{s}{ }^{2}$ is the variance of stego, and $\sigma_{c s}$ is covariance of cover as well as stego.

- Mean and standard deviation: In probability and statistics, the mean [56] and expected value synonymously refer to one particular measure of the central tendency. Also, this pertains to either of a probability distribution or random variable, which is characterized by the specific distribution. Also, we may say that the standard deviation [57] or " $\sigma$ " is the measured square root of the variance of " $X$." Rather it is actually the square root of the estimated average value of " $(X-\mu)^{2}$."

- Entropy: Here, the term "message” may stand for any event, character, or sample, which is drawn from specific distribution of data stream. Thus "entropy" [58] is known to characterize our uncertainty regarding the source of information. Since entropy is greater for more random sources, so it is understood as the measure of uncertainty instead of certainty. Entropy may be defined in some context of any probabilistic model. We may say that independent fair coin flips always have some entropy of 1 bit each per flip. Considering a source generating one long string of character B's, it will always have entropy of 0 . This is because the next character here will always be a "B." The next following points are to be considered:

- The net amount of existing entropy might not always be some integer number of the bits.

- Some data bits might even not convey any information. As an example, some data structures sometimes redundantly store information. Also regardless of the specific information residing in the very data structure, they might have identical sections. Given any data source, it gives the average of the bits that are needed for encoding it. 
- Skewness and kurtosis: In case of any nonparametric skew, we can define it as $(\mu-v) / \sigma$. Here, $\mu$ is its mean, $v$ is its median, and $\sigma$ is its standard deviation. In cases where distribution remains symmetric, the mean becomes equal to median. Thus, such distribution will always have zero skewness [59]. Kurtosis [60] determines how sharp and tall the central peak could be when it is relative to some standard bell curve. Also, there are several interpretations for kurtosis, along with those how certain measures have to be interpreted. The primary measures are tail weight, peakedness (i.e., width of peak), and also lack of shoulders (i.e., when distribution primarily resides in peak and tails and not in between).

Consider some univariate data $D_{1}, D_{2}, \ldots, D_{N}$, then their skewness as well as kurtosis is found as follows:

$$
\begin{gathered}
\text { Skewness }=\frac{\sum_{i=1}^{N}\left(D_{i}-\mu\right)^{3} / N}{\sigma^{3}} \\
\text { Kurtosis }=\frac{\sum_{i=1}^{N}\left(D_{i}-\mu\right)^{4} / N}{\sigma^{4}}
\end{gathered}
$$

where $\mu$ is mean, $\sigma$ is standard deviation, and $N$ is number of pixels.

\subsection{Conclusion}

The rapid sprout in the usage of sensitive information exchange through the Internet or any public platform causes a major security concern in these days. More essentially, digital data has given an easy access to communication of its content that can also be copied without any kind of degradation or loss. Therefore, the urgency of security during global communication is obviously quite palpable nowadays. Hence, the data hiding in the seemingly unimportant cover medium is perpetuated. The realm of steganography ratifies the stated fact to safeguard the privacy of data. Unlike cryptography, steganography brings forth various techniques that strive to hide the existence of any hidden information along with keeping it encrypted. On the other hand, any apparently visible encrypted information is definitely more likely to captivate the interest of some hackers and crackers. Therefore, precisely saying, cryptography is a practice of shielding the very contents of the cryptic messages alone. On the other hand, steganography is seriously bothered with camouflaging the fact that some confidential information is being sent, along with concealing the very contents of the message. Hence, using steganographic procedures in the field of big data enhances their security. 


\section{References}

[1] Mukherjee, S., \& Sanyal, G. A chaos based image steganographic system", Multimed Tools Appl, Springer, 2018, 77 (21).

[2] Gupta, B., Agrawal, D.P., \& Yamaguchi, S. Handbook of Research on Modern Cryptographic Solutions for Computer and Cyber Security, 2016.

[3] Saha, PK., Strand, R., \& Borgefors, G. Digital Topology and Geometry in Medical Imaging: A Survey. IEEE Transactions on Medical Imaging, 2015, 34(9), 1940-1964.

[4] Potdar, V., \& Chang, E. Gray level modification steganography for secret communication, IEEE International Conference on Industrial Informatics, Berlin, Germany, 2004, 355-368

[5] Dagadita, MA., Slusanschi, El., \& Dobre, R. Data Hiding Using Steganography. 12th International Symposium on Parallel and Distributed Computing, IEEE, 2013, 159-166

[6] Katzenbeisser, S., \& Petitcolas, F. A. Information Hiding. Artech House information security and privacy series, Artech House, 2015, ISBN 978-1-60807-928-5, pp. I-XVI, 1-299

[7] Mukherjee, S., \& Sanyal, G. (2018): A Multi-level Image Steganography Methodology Based on Adaptive PMS and Block Based Pixel Swapping, Multimed Tools Appl, Springer, 2018

[8] Mukherjee, S., \& Sanyal, G. Extended Power Modulus Scrambling (PMS) Based Image Steganography with Bit Mapping Insertion. In: Fahrnberger G., Gopinathan S., Parida L. (eds) Distributed Computing and Internet Technology, 2019, ICDCIT 2019. Lecture Notes in Computer Science, vol 11319. 364-379. Springer, Cham

[9] Mukherjee, S., Roy, S., \& Sanyal, G. Image Steganography Using Mid Position Value Technique, International Conference on Computational Intelligence and Data Science (ICCIDS), Procedia Computer Science, 2018, 132,461-468, Elsevier

[10] Mukherjee, S., \& Sanyal, G. A Novel Image Steganography Methodology Based on Adaptive PMS Technique. In: Sa P., Sahoo M., Murugappan M., Wu Y., Majhi B. (eds) Progress in Intelligent Computing Techniques: Theory, Practice, and Applications. Advances in Intelligent Systems and Computing, 2018, vol 518. 157-164. Springer, Singapore.

[11] Das, Shantanu, Tixeuil, Sebastien (Eds.). Structural Information and Communication Complexity. 24th International Colloquium, SIROCCO 2017, Porquerolles, France, 2017

[12] 6. Mukherjee, S., Ash, S., \& Sanyal, G. A Novel Differential Calculus Based Image Steganography with Crossover, International Journal of Information and Communication Engineering, World Academy of Science, Engineering and Technology (WASET), (2015), 9(4): 1056-1062

[13] Zhengan, H., Shengli, L., Xianping, M., Kefei, C., \& Jin, L. Insight of the Protection for Data Security Under Selective Opening Attacks. Information Sciences, 2017, 412-413, 223-241

[14] Mukherjee, S., \& Sanyal, G. Enhanced Position Power First Mapping (PPFM) based Image Steganography, International Journal of Computers and Applications (IJCA), Taylor and Francis, 2017, 39 (2): 59-68,

[15] Mukherjee, S., \& Sanyal, G. Edge Based Image Steganography with Variable Threshold, Multimed Tools Appl, Springer, 2018.

[16] Khosla, S., \& Kaur, P. Secure Data Hiding Technique using Video Steganography and Watermarking. International Journal of Computer Applications, 2014, 95(20), 7-12.

[17] Mukherjee, S., \& Sanyal, G. A Physical Equation Based Image Steganography with Electro-magnetic Embedding, Multimed Tools Appl, Springer, 2019

[18] Kaminsky, Alan., Kurdziel, Michael., \& Radziszowski, Stanislaw. An Overview of Cryptanalysis Research for the Advanced Encryption Standard. Proceedings - IEEE Military Communications Conference MILCOM, 2010, 10.1109/MILCOM.2010.5680130.

[19] Khalaf, Abdulrahman. Fast Image Encryption based on Random Image Key. International Journal of Computer Applications, 2016, 134. 
[20] Dai, Hong-Ning., Wang, Qiu., Dong, Li., \& Wong, Raymond. On Eavesdropping Attacks in Wireless Sensor Networks with Directional Antennas. International Journal of Distributed Sensor Networks, 2013, 2013.

[21] Panda, M., \& Nag, A. Plain Text Encryption Using AES, DES and SALSA20 by Java Based Bouncy Castle API on Windows and Linux. 2015 Second International Conference on Advances in Computing and Communication Engineering, 2015, 541-548.

[22] Wei, S., Sun, Z., Yin, R., \& Yuan, J. Trade-Off Between Security and Performance in Block Ciphered Systems With Erroneous Ciphertexts. IEEE Transactions on Information Forensics and Security, 2013, 8, 636-645.

[23] Khalaf, Abdulrahman. Fast Image Encryption based on Random Image Key. International Journal of Computer Applications, 2016, 134.

[24] Ping, L., Jin, L., Zhengan, H., Tong, L., Chong-Zhi, G., Siu-Ming, Y., \& Kai, C. Multi-Key PrivacyPreserving Deep Learning in Cloud Computing. Future Generation Computer Systems, 2017, 74, 76-85.

[25] Muhammad, K., Ahmad, J., Rho, S., \& Baik, S.W. Image steganography for authenticity of visual contents in social networks. Multimedia Tools and Applications, 2017, 76, 18985-19004.

[26] Sobti, Rajeev., \& Ganesan, Geetha. Cryptographic Hash Functions: A Review. International Journal of Computer Science Issues, 2012, 9, 461-479.

[27] Sedighi, V., Cogranne, R., \& Fridrich, J. Content-Adaptive Steganography by Minimizing Statistical Detectability.: IEEE Transactions on Information Forensics and Security, 2016, 11(2), 221-234

[28] Steendam, H. On the Selection of the Redundant Carrier Positions in UW-OFDM. IEEE Transactions on Signal Processing, 2013, 61(5), 1112-1120.

[29] Zhu, L., \& Zhu, L. Electronic signature based on digital signature and digital watermarking. 5th International Congress on Image and Signal Processing, CISP, 2012, 1644-1647

[30] Zhang, Weiming., Zhang, Xinpeng., \& Wang, Shuozhong., "Near-Optimal Codes for Information Embedding in Gray-Scale Signals," IEEE Transactions on Information Theory, 2010, 1262-1270.

[31] Abdallah, E.E., Ben Hamza, A., \& Bhattacharya, P. MPEG Video Watermarking Using Tensor Singular Value Decomposition, International Conference Image Analysis and Recognition, ICIAR 2007: Image Analysis and Recognition, pp. 772-783

[32] Li, J., Yu, C., Gupta, BB. et al. Color image watermarking scheme based on quaternion Hadamard transform and Schur decomposition. Multimed Tools Appl, 2018, 77(4), 4545-4561.

[33] Do, Q., Martini, B., \& Choo K-K, R. The Role of the Adversary Model in Applied Security Research. Computers \& Security

[34] Kahn, D. The History of Steganography. Lect Notes Comput Sci, 1996, 1174. 1-5.

[35] Siper, A., Farley, R., \& Lombardo, C. The Rise of Steganography. Proceedings of Student/ Faculty Research Day, CSIS, Pace University, D1_1-7, 2005.

[36] Hamid, Nagham., Yahya, Abid., Ahmad, R. Badlishah., Osamah, M. Al-Qershi., Alzubaidy, Dheiaa Aldeen Najim., \& Kanaan, Lubna. Enhancing the Robustness of Digital Image Steganography Using ECC and Redundancy. Journal of Information Science and Engineering, 2012.

[37] Carson, Austin., \& Yarhi-Milo, Keren., Covert Communication: The Intelligibility and Credibility of Signaling in Secret, Security Studies, 2016, 26, 124-156.

[38] Hussain, A., Al-Fayadh, A., \& Radi, N. Image Compression Techniques: A Survey in Lossless and Lossy algorithms, Neurocomputing, 2018, 300, 44-69

[39] Borges, PVK., Mayer, J., \& Izquierdo, E. Robust and Transparent Color Modulation for Text Data Hiding. IEEE Transactions on Multimedia, 2008, 10(8), 1479-1489. 
[40] Cem Kasapbaşi, M., \& Elmasry, W. New LSB-based colour image steganography method to enhance the efficiency in payload capacity, security and integrity check. Sādhanā (2018) 43: 68.

[41] Febryan, A., Purboyo, TW., \& Saputra, RE. Steganography Methods on Text, Audio, Image and Video: A Survey. International. Journal of Applied Engineering Research, 2017, 12(21), 10485-10490

[42] Ahvanooey, MT., Li, Q., Hou, J., et al. AlTSteg: An Innovative Text Steganography Technique for Hidden Transmission of Text Message via Social Media. IEEE Access, 2018, 6, 65981-65995.

[43] Khalil, M., Li, JP., \& Kumar, K. (2015): Color constancy models inspired by human visual system: Survey paper. 12th International Computer Conference on Wavelet Active Media Technology and Information Processing. 432-435

[44] Zhang, Y-M., \& Cen, J-J. (2010) Research on method of transformation from bitmap to vector graphics based on Adobe Illustrator CS4. International Conference on Advanced Computer Theory and Engineering, IEEE, V3_75-77

[45] Olugbara, 00., Adetiba, E., \& Oyewole, SA. Pixel Intensity Clustering Algorithm for Multilevel Image Segmentation. Mathematical Problems in Engineering, 2015, 1-19

[46] Hashim, M., Mohd, R., \& Alwan, A. A review and open issues of multifarious image steganography techniques in spatial domain. Journal of Theoretical and Applied Information Technology, 2018, 96(4). 956-977

[47] Elham, Ghasemi., Shanbezadeh, Jamshid., \& Nima, Fassihi. High Capacity Image Steganography using Wavelet Transform and Genetic Algorithm. Lecture Notes in Engineering and Computer Science, 2011, 1. 10.1007/978-1-4614-1695-1_30.

[48] Yang, C., Weng, C., Wang, S., et al Adaptive Data Hiding in Edge Areas of Images With Spatial LSB Domain Systems. IEEE Transactions on Information Forensics and Security, 2008, 3, 488-497

[49] Shen, S., \& Huang, L. A Data Hiding Scheme Using Pixel Value Differencing and Improving Exploiting Modification Directions. Computers and Security, 2014, 48, 131-141

[50] Dey, N., Roy, A. B., \& Dey, S. A novel approach of color image hiding using RGB color planes and DWT. International Journal of Computer Applications, 2012, 36(5), 19-24.

[51] Zhou, X., Yunhao Bai, Y., \& Wang, C. Image Compression Based on Discrete Cosine Transform and Multistage Vector Quantization, International Journal of Multimedia and Ubiquitous Engineering, 2015, 10(6), 347-356

[52] Bhattacharyya, D., \& Kim, T. Image Data Hiding Technique Using Discrete Fourier Transformation. In: Kim T., Adeli H., Robles R.J., Balitanas M. (eds) Ubiquitous Computing and Multimedia Applications. Communications in Computer and Information Science, Springer, Berlin, Heidelberg, 2011, 151

[53] Hansen, B. The Integrated Mean Squared Error of Series Regression and a Rosenthal HilbertSpace Inequality. Econometric Theory, 2015, 31, 337-361

[54] Tao, D., Di, S., Liang, X., Chen, Z., \& Cappello, F. Fixed-PSNR Lossy Compression for Scientific Data. 2018 IEEE International Conference on Cluster Computing (CLUSTER), 2018, 314-318.

[55] Dosselmann, R., \& Yang, X.D. A comprehensive assessment of the structural similarity index. Signal, Image and Video Processing. SIViP. Vol. 5, pp 81-91 (2011)

[56] Malik, F. Mean and Standard Deviation Features of Color Histogram Using Laplacian Filter for Content-Based Image Retrieval. Journal of Theoretical and Applied Information Technology, 2011, 34

[57] Sung, Jungmin., Kim, Dae-Chul., Choi, Bong-Yeol., \& Ha, Yeong-Ho. Image Thresholding Using Standard Deviation. Proceedings of SPIE - The International Society for Optical Engineering, 2014, 9024. 10.1117/12.2040990. 
[58] Duncan, K., \& Sarkar, S. (2012) Relational Entropy-Based Saliency Detection in Images and Videos. 19th IEEE International Conference on Image Processing. 1093-1096

[59] Koo, H., \& Cho, N. Skew Estimation of Natural Images Based on a Salient Line Detector. J. Electronic Imaging, 2013, 22.

[60] Ferzli, R., Girija, L., \& Ali, W. (2010) Efficient Implementation of Kurtosis Based No Reference Image Sharpness Metric. in Jaakko Astola \& Karen O. Egiazarian (Ed.): Proc. SPIE 7532, Image Processing: Algorithms and Systems VIII 
